\title{
Current Understanding of the Cellular Biology and Molecular Structure of the Antidiuretic Hormone-stimulated Water Transport Pathway
}

\author{
H. William Harris, Jr.," Kevin Strange, ${ }^{*}$ and Mark L. Zeidel*\$s \\ Divisions of Nephrology, Children's Hospital,* Brigham and Women's Hospital, ${ }^{\ddagger}$ and West Roxbury \\ Veterans Administration Hospital, $\$$ Boston, Massachusetts 02115
}

\begin{abstract}
Introduction
The ability of the kidney to conserve or excrete free water independent of changes in solute excretion was a prerequisite for the successful adaptation of vertebrates to prolonged periods on land $(1,2)$. Renal conservation of water in amphibia, reptiles, birds, and mammals is controlled by antidiuretic hormone $(\mathrm{ADH}){ }^{1,2}$ In these animals, body fluid osmolality is maintained within very narrow limits. When body fluid osmolality decreases, ADH levels fall, causing the excretion of free water via a hypotonic urine. In contrast, when body fluid osmolality rises, $\mathrm{ADH}$ is released, stimulating free water retention and excretion of a hypertonic urine. ADH controls renal water retention by inserting water channels into the normally watertight apical membranes of the collecting duct. This in turn allows water flow from the tubule lumen along osmotic gradients between the tubular fluid and the surrounding interstitium (2). In addition, ADH enhances sodium reabsorption by the collecting duct (2) and, in some animals, increases transepithelial solute transport in the medullary thick ascending limb, thereby increasing osmotic gradients for water reabsorption. In certain amphibia, dilute urine is stored in a large bladder where it can be reabsorbed in response to ADH secreted when the body fluid osmolality rises (1). Since ADH-responsive properties of toad urinary bladder granular epithelial cells resemble closely those of principal cells of the mammalian collecting duct, the toad bladder is a useful model of $\mathrm{ADH}$-responsive renal epithelia.

In this review, we will discuss our current understanding of the cellular biology and molecular structure of the ADH water
\end{abstract}

Address reprint requests to Dr. Harris, Division of Nephrology, Children's Hospital, 300 Longwood Avenue, Boston, MA 02115.

Received for publication 11 April 1991.

1. Abbreviations used in this paper: $\mathrm{ADH}$, antidiuretic hormone; $C C D$, cortical collecting duct; $E_{A}$, activation energy; FMA, fluorescein mercuric acetate; HRP, horseradish peroxidase; MVB, multivesicular body; pCMBS, para-chloromercuribenzene sulfonic acid; $P_{D}$, diffusional water permeability; $P_{f}$, osmotic water permeability; $p_{r}$ gramicidin channel permeability; WCV, water channel-containing vesicle. 2. In this article antidiuretic hormone (ADH) is used instead of the term vasopressin in an effort to emphasize the effects of this hormone on renal epithelial cells.

J. Clin. Invest.

(c) The American Society for Clinical Investigation, Inc. 0021-9738/91/07/001/08 $\$ 2.00$

Volume 88, July 1991, 001-008 channel. In particular, we will highlight recent research developments of several of the most basic aspects of this complex and highly conserved membrane transport system. In accordance with space limitations, we have focused our discussion of this subject and attempted to point out important areas of uncertainty as well as anticipated future developments.

The apical membranes of unstimulated ADH-responsive epithelial cells possess an unusually low water permeability In the absence of ADH stimulation, epithelial cells of the toad urinary bladder exhibit a strikingly low osmotic water permeability $\left(P_{f}\right)$ of $\sim 2 \times 10^{-4} \mathrm{~cm} / \mathrm{s}(3)$. Similarly, the $P_{f}$ of unstimulated cortical and medullary collecting ducts is also low ranging from 15 to $90 \times 10^{-4} \mathrm{~cm} / \mathrm{s}(2,4,5)$ (Table I). Initial studies (reviewed in Finkelstein [3]) suggesting that the apical membrane of epithelial cells represents the major permeability barrier to water have been confirmed by direct measurements of both apical and basolateral $P_{f}$ in selected epithelial cells. In the absence of ADH stimulation, apical membrane $P_{f}$ is low in both principal cells of the rabbit cortical collecting duct (CCD) $\left(20 \times 10^{-4} \mathrm{~cm} / \mathrm{s}\right)$ and granular cells of toad urinary bladder (reviewed in Harris and Handler [6]). The somewhat higher basal $P_{f}$ exhibited by mammalian collecting duct as compared to the toad bladder may be due to the presence of a few apical membrane water channels in the former even in the absence of $\mathrm{ADH}$ (7). In contrast, $P_{f}$ values for the principal cells' basolateral membrane are in excess of $70 \times 10^{-4} \mathrm{~cm} / \mathrm{s} \mathrm{(4).} \mathrm{This} \mathrm{higher}$ $P_{f}$ coupled with the fact that the surface area of the basolateral membrane of an individual principal cell is at least 7 times larger than that measured for the apical membrane means that the basolateral membrane $P_{f}$ is 27 times larger than that of the apical membrane (4).

\section{Structural features of apical membrane that may determine the low $P_{f}$ exhibited by $A D H$-responsive cells in the basal state}

The exact mechanisms by which ADH-responsive epithelial cells maintain their low apical membrane $P_{f}$ remain unclear and are under active investigation (8). Interestingly, in addition to a low $P_{f}$, the apical membranes of inner medullary collecting duct as well as toad bladder epithelial cells have all recently been shown to be relatively impermeable to $\mathrm{NH}_{3}(9)$. The combination of the low $\mathrm{P}_{\mathrm{f}}$ and $\mathrm{NH}_{3}$ permeabilities suggests that these apical membranes may share highly unusual structural features such as an unusual lipid composition or unique arrangement of lipids within the lipid bilayer. Alterations in membrane lipid composition of both artificial lipid bilayers (4, 10) and human erythrocytes (11) cause changes in the $P_{f}$ 's of 
Table I. Characteristics of Water Flow through Apical Membranes of ADH-responsive Epithelial Cells

\begin{tabular}{lcc}
\hline & $\begin{array}{c}\text { No ADH } \\
\text { (no ADH water channels) }\end{array}$ & $\begin{array}{c}\text { DH stimulation } \\
\text { (ADH water channels) }\end{array}$ \\
\hline $\mathrm{P}_{\mathrm{f}}(\mathrm{cm} / \mathrm{s})$ & $2-90 \times 10^{-4}$ & $210-900 \times 10^{-4}$ \\
$\mathrm{P}_{\mathrm{f}} / \mathrm{P}_{\mathrm{D}}$ & 1 & $9-17$ \\
$\mathrm{E}_{\mathrm{A}}\left(\mathrm{kcal} / \mathrm{mol} \mathrm{per}^{\circ} \mathrm{K}\right)$ & $10-15$ & $2-5$ \\
Response to mercurials & None & Inhibit water flow \\
Proton permeability & Low & Increased \\
\hline
\end{tabular}

these membranes. In addition to membrane lipid composition, the distribution of lipids within the bilayer may also determine $P_{f}$. Since it now appears that tight junctions between epithelial cells permit free movement of lipid substituents between basolateral and apical domains of cytoplasmic but not exoplasmic leaflets (12), it is likely that the distinctive permeability properties of the apical membrane are conferred by the substituents in its exoplasmic leaflet. Membrane permeability measurements combined with protein and lipid composition analysis from recently isolated preparations of highly purified apical membranes from toad urinary bladder (8) may offer insights into which of these mechanisms is responsible for its unusually low permeability properties.

\section{Characteristics of the ADH-elicited increase in water permeability}

$\mathrm{ADH}$ binds to specific $\mathrm{V} 2$ receptors activating a variety of signal transduction pathways including cAMP (13). Within minutes, $\mathrm{ADH}$ stimulation causes increases in the apical membrane $P_{f}$ of 4-100-fold. Values of ADH-stimulated apical membrane $P_{f}$ are found variously at $210 \times 10^{-4} \mathrm{~cm} / \mathrm{s}$ in toad urinary bladder (4), $90 \times 10^{-4} \mathrm{~cm} / \mathrm{s}$ in rabbit $\mathrm{CCD}(5)$, and 900 $\times 10^{-4} \mathrm{~cm} / \mathrm{s}$ in rat $\mathrm{CCD}$ after prolonged $\mathrm{ADH}$ stimulation (14) (Table I). In a similar fashion, ADH stimulation causes inner medullary collecting duct $P_{\mathrm{f}}$ to rise to values between 150 and $700 \times 10^{-4} \mathrm{~cm} / \mathrm{s}$ depending on the segment being studied $(2,5)$.

Early research efforts postulated that ADH increased $\mathrm{P}_{\mathrm{f}}$ and permeabilities to small nonelectrolytes by changing apical membrane lipid components. However, more recent work clearly indicates that $\mathrm{ADH}$ increases apical membrane $\mathrm{P}_{\mathrm{f}}$ by insertion of unique water channels (reviewed in Finkelstein [3]). Evidence supporting the existence of ADH water channels can be grouped into three major areas. First, the permeability changes elicited by ADH are relatively selective for water. ADH stimulation causes only modest increases in the permeabilities to small nonelectrolytes as compared with its dramatic enhancement in $P_{f}$. If $A D H$ enhanced $P_{f}$ via a generalized increase in the apical membrane fluidity, studies in artificial membranes predict that permeabilities to water and small nonelectrolytes would increase to a similar degree (3). Secondly, a large number of ultrastructural studies have identified structures called membrane particle aggregates (particle aggregates) whose appearance in the apical membrane correlates closely with the presence, magnitude, and kinetics of the ADH-elicited increase in apical membrane $P_{f}$ (see below). Thirdly, biophysical studies of ADH-elicited water flow are best explained not by the permeation of water through the lipid bilayer, but by water permeation through aqueous pores or channels (3). The perme- ation of water across pure lipid bilayers exhibits several distinct characteristics including an activation energy $\left(E_{A}\right)$ of $10-15$ $\mathrm{kcal} / \mathrm{mol}$, insensitivity to preincubation with mercurial reagents such as parachloromercuribenzene sulfonic acid (pCMBS) and $\mathrm{HgCl}_{2}$, and ratios of osmotic water permeability/diffusional water permeability $\left(P_{f} / P_{D}\right)$ approaching 1 (3). Water flow in unstimulated toad urinary bladders exhibits a similar pattern with an $\mathrm{E}_{\mathrm{A}}$ of $10-15 \mathrm{kcal} / \mathrm{mol}$ and insensitivity to exposure to mercurials (Table $I$ ). The $P_{f} / P_{D}$ ratio is difficult to measure accurately owing to the exceptionally low water permeability of this preparation. In contrast, minutes after $\mathrm{ADH}$ stimulation of toad bladders, the $\mathrm{E}_{\mathrm{A}}$ of water flow is 3 $\mathrm{kcal} / \mathrm{mol}(15)$ and is markedly inhibited by mercurial reagents (16). In studies that account carefully for unstirred layer effects, estimates of $P_{f} / P_{D}$ range from 9 to $17(17)$. These values are also similar to those obtained by measurement of water permeation through the aqueous channel formed by the intercalating antibiotic gramicidin (3) or the human erythrocyte water channel (11). Qualitatively similar results have been obtained in CCD (7). However, the accuracy and meaning of these criteria have been difficult to interpret in intact epithelia because of the combination of the presence of significant unstirred layer effects leading to overestimates of $P_{f} / P_{D}$ and because temperature changes (necessary to examine $\mathrm{E}_{\mathrm{A}}$ ) influence all cellular metabolic and structural processes, and not just those present in apical membrane $P_{f}(3,7,15,17)$. Despite these technical and theoretical difficulties, these results have been accepted as biophysical evidence supporting the existence of $\mathrm{ADH}$ water channels.

\section{Vesicles deliver and remove ADH water channels from the} apical membranes of $A D H$-responsive cells

It is now generally believed that $\mathrm{ADH}$ controls the movement of functional water channels between the apical membrane and the cytoplasm (Fig. 1). This so-called "membrane shuttling hypothesis" (18) had its origins in early work by numerous investigators using freeze-fracture electron microscopy to examine morphological changes in the apical membranes of ADH-stimulated cells (reviewed in detail in Harris and Handler [6]). These included the anuran urinary bladder, toad skin, and mammalian collecting duct. Use of this technique has established the existence of a linear relationship between the number of apical membrane particle aggregates and tissue $P_{\mathrm{f}}$. These data have led to the suggestion that particle aggregates, which are presumably proteins, are either ADH water channels themselves or are closely associated with the structures responsible for transmembrane water flow.

A series of ultrastructural studies (18-20) revealed that before ADH stimulation toad bladder particle aggregates are located as parallel linear arrays in the limiting membranes of large (up to $1 \mu \mathrm{m}$ long), tubular, subapical intracellular vesicles termed "aggrephores" (20). A combination of morphological (20) and tissue capacitance (21) measurements has demonstrated that ADH stimulation causes a $10-30 \%$ increase in apical membrane surface area with a time course paralleling the rise in tissue $P_{f}$. In a similar fashion, termination of ADH stimulation results in a prompt decrease in tissue $P_{f}$ and concomitant retrieval of granular cell apical membrane in the form of endocytic vesicles $(6,18,20,22)$ containing particle aggregates (23).

More recent work has validated other key aspects of the shuttle hypothesis. Upon their retrieval from the apical mem- 


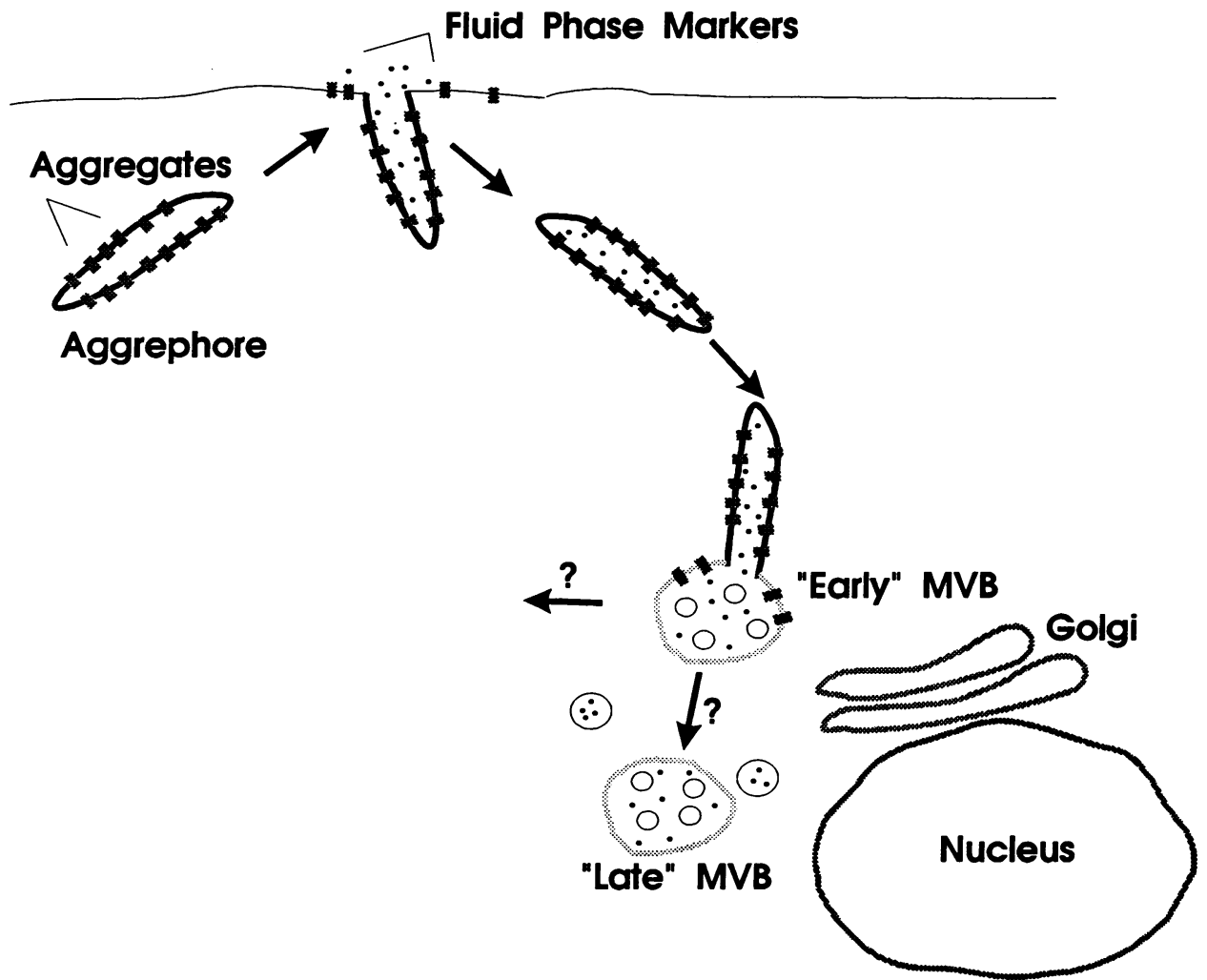

Figure 1. Schematic overview of apical membrane insertion and retrieval elicited by ADH in the granular cell of the toad urinary bladder. As first proposed by Wade et al. (18) in the shuttle hypothesis, vesicles containing large numbers of ADH water channels (aggrephores) fuse with the granular cell apical membrane which possesses low $P_{f}$. Aggrephores insert water channels into the apical membrane accounting for the large selective increase in apical membrane $P_{f}$ within minutes of ADH stimulation. A morphological marker for this water channel-containing membrane are structures called particle aggregates that remain in fused aggrephores as well as translocate into the apical membrane itself. Reduction in apical membrane $P_{f}$ occurs by retrieval of WCV that sequester fluid phase markers within their lumens upon endocytosis. A combination of morphological (see references 23 and 40) and functional (see references 24-26 and 41) data show that functional water channels and particle aggregates are first contained within subapical tubular and spherical vesicles. However, with time, both vesicle contents and water channel containing membrane appear to be transferred to another endosomal vesicle termed the multivesicular body (MVB). Initially, early MVBs contain both fluid phase markers and particle aggregates. $60 \mathrm{~min}$ after the initiation of apical membrane retrieval functional water channels are no longer found in MVBs. Question marks indicate that it is presently unknown whether water channel membrane recycles or whether there is sorting of membrane in MVBs.

brane, endocytic vesicles entrap fluid phase markers contained in the solution bathing the apical membrane which serve to distinguish retrieved vesicles from others present within the cell (Fig. 1). These retrieved vesicles containing either entrapped carboxyfluorescein, fluorescein dextran ( $\mathrm{F}$ dextran), or horseradish peroxidase (HRP) have been recovered from homogenates of granular cells $(6,24-28)$ and the renal papillas of Brattleboro rats (29) and used to measure vesicle membrane $P_{f}$ and proton permeability $\left(\mathrm{P}_{\mathrm{H}^{+}}\right)$. The $\mathrm{P}_{\mathrm{f}}$ of these water channel-containing vesicles (WCV) is extraordinarily high, demonstrating that functional water channels are removed from the apical membrane by vesicle endocytosis. Similar membrane shuttling mechanisms are thought to be important in controlling proton (30), calcium (31), sodium (32), and glucose (33) transport in a number of cell types.

Control of WCV endocytosis and intracellular sorting of water channel containing membrane are areas under active investigation. Several observations suggest that endosomes containing water channels do not acidify their luminal contents. This conclusion is based on data showing that $(a)$ there is no luminal acidification of WCV in vitro after addition of ATP $(26,27,34),(b)$ measurements of these vesicles in situ using the 3-(2,4-dinitroanilino)-3'-amino- $n$-methyldipropylamine (DAMP) method show their lumens are not acidic (6), and $(c)$ immunoblots of purified WCV proteins with anti-proton pump antisera reveal these vesicles do not contain the major subunits of the $\mathrm{H}^{+}$ATPase necessary for activity (35). Factors that modulate retrieval of water channels include ADH removal $(6,18,20)$, the magnitude of a transepithelial osmotic gradient $(22,27,36)$, and cytoplasmic dilution or cell swelling (37). Water channel vesicle endocytosis has recently been associated with changes in the phosphorylation state of a vesicle-associated $15.5-\mathrm{kD}$ protein (38). Investigation of the intracellular sorting of water channel-containing membrane has been hampered by lack of specific water channel membrane markers. However, studies in the toad bladder using HRP have demonstrated clearly that particle aggregates are initially localized to large tubular vesicles which are presumably retrieved aggrephores $(23,39)$ and contain functional water channels (WCV) (see above).

Recently, the intracellular sorting of fluid phase markers and particle aggregates has been studied in detail by Coleman and Wade (40). Their studies in the toad bladder show that within $30 \mathrm{~min}$ after the initiation of endocytosis, the endocytosed HRP appears within the lumens of other morphologically distinct endocytic vesicles called multivesicular bodies (MVBs) located in the perinuclear region of the granular cell cytoplasm (Fig. 1). These "early" MVBs contain well-organized particle aggregates and lack the lysosomal marker, acid phosphatase. $60 \mathrm{~min}$ after the initiation of water channel retrieval, HRP is located in "late" MVBs that possess either few or no particle aggregates and contain acid phosphatase. At this time, vesicles containing particle aggregates but no HRP appear in the vicinity of these "late" MVBs. Measurements of late MVBs indicate that their limiting membranes do not contain large numbers of functional water channels since minimum 
values for their $P_{f}$ and $P_{H^{+}}$are only $1 \%$ and $5 \%$, respectively, of the values exhibited by WCV (41). Taken together, these results suggest that water channels retrieved from the granular cell apical membrane separate from fluid phase markers such as HRP sequestered within endocytic vesicles. In this regard, early MVBs may function as a sorting organelle, possibly allowing the recycling of at least some water channels for later reuse in a subsequent $\mathrm{ADH}$ stimulation.

Vesicle systems that deliver and sort water channels may be different in various tissues. In addition to aggrephores, other aggregate transport organelles have been described. Ding et al. (39) observed aggrephore-like structures in the frog urinary bladder that did not fuse with the apical membrane during ADH stimulation. Instead, particle aggregates appear to be delivered via spherical, possibly coated vesicles with variable diameters. Particle aggregate-containing vesicles with the morphology of aggrephores are either rare or absent in cells of the mammalian collecting duct (6). The vesicle structures involved in the insertion of particle aggregates in mammalian collecting duct are currently unknown. Brown and Orci (42), however, have demonstrated that particle aggregates of the rat collecting duct are located in apical membrane-coated pits. Present evidence suggests that coated vesicles are involved in retrieval of apical membrane water channels $(42,43)$. Fluid phase markers contained within the lumens of these vesicles are first transferred to endosomes and ultimately enter lysosomes (43).

The mechanisms by which aggregate-containing organelles move to, fuse with, and are retrieved from the apical membrane are unclear. A good deal of indirect evidence, however, has implicated the cytoskeleton in this process. Numerous studies using colchicine, cytochalasin B, and thin-section electron microscopy suggest that both microtubules and microfilaments participate in vesicle-mediated particle aggregate insertion and removal (reviewed in Pearl and Taylor [44]). Their exact roles however remain to be determined. Aggrephore translocation also may involve mechanochemical transduction events such as those required for the movement of other types of organelles (45). If this is true, then microtubules might function as "tracks" on which the aggrephores are moved to the apical membrane by molecular motors such as dynein or kinesin (reviewed in Vale [45]). In support of this hypothesis, several investigators have shown that inhibitors of dynein ATPase activity also inhibit ADH-sensitive water flow (44).

\section{Properties of the ADH-elicited water channel inferred from} biophysical studies

Biophysical techniques have also provided insight into likely structural features of the ADH water channel. These data have been obtained from a combination of intact epithelia and vesicle preparations containing ADH water channels (WCV) isolated from both the toad urinary bladder (24-27) and rat medulla $(29,34)$. These vesicle studies are particularly important since they have examined water channel characteristics in the absence of confounding variables imposed by intact epithelia as discussed above. The $\mathrm{P}_{\mathrm{f}}$ of WCV is extraordinarily high $(0.1-$ $0.2 \mathrm{~cm} / \mathrm{s}$ ). If we assume that the $P_{f}$ of an individual $A D H$ water channel contained in toad bladder WCV is similar to that of gramicidin pores $\left(p_{\mathrm{f}}=3 \times 10^{-14} \mathrm{~cm}^{3} / \mathrm{s}\right)$, then the density of water channels per unit membrane area in these vesicles is $P_{f} / p_{f}$ or $5 \times 10^{4}$ channels $/ \mu \mathrm{m}^{2}$. This value is 10 times higher than that calculated for the human erythrocyte membrane $(3,11)$ and is similar to the density of acetylcholine receptors in the postsynaptic membranes of Torpedo californica (46). These considerations suggest that WCV contain ADH water channels at an extraordinarily high density. A high density of water channels suggests that water channel proteins are a major component of the total protein content of WCV (see below). Data from intact epithelia as well as these WCV demonstrate that ADH water channels are selective for water, excluding small nonelectrolytes such as acetamide and urea $(3,17,27)$. These considerations indicate that at some point along its course the ADH water channel must be very narrow, with a diameter of $\sim 2 \AA$ (3).

The high selectivity of the ADH water channel suggests that water molecules traverse the channel in a single-file fashion (3, 17). If single file transport occurs, the $P_{f} / P_{D}$ ratio determines the number of water molecules in the pore at any given time. $P_{f} / P_{D}$ ratios are difficult to assess directly because studies in tight epithelia are complicated for reasons stated above. This is combined with the fact that the $P_{f}$ exhibited by WCV is so large that it precludes accurate determinations of vesicle $P_{D}(24,25)$. Together these considerations have made it difficult to relate the apparent $P_{f} / P_{D}$ ratios exhibited by $A D H$ water flow to actual characteristics of $\mathrm{ADH}$ channel structure.

Mercurial inhibition of $\mathrm{ADH}$ water flux indicates that sulfhydryl groups may be critical to water channel function (16, $26,47)$. When a combination of mercurials and sulfhydryl protection agents such as mercaptoethanol is added to either intact epithelia or WCV, no inhibition of ADH water flow is observed $(16,25)$.

The ability of water to traverse the ADH water channel suggests that the channel might also be permeable to the hydronium ion $\left(\mathrm{H}_{3} \mathrm{O}^{+}\right)$or, alternatively, that protons cross the channel by sequential association-dissociation with water molecules within the channel. This concept is supported by data showing that narrow channels such as the gramicidin pore (3) and artificial water channels (48) have large proton permeabilities. In addition, ADH stimulation increases transepithelial proton permeability in the toad bladder (49). Measured proton permeability values $\left(\mathrm{P}_{\mathrm{H}^{+}}\right)$for WCV from the toad bladder range from 5 to $50 \times 10^{-3} \mathrm{~cm} / \mathrm{s}(26,27)$. The fact that $P_{\mathrm{H}^{+}}$in these preparations exhibits an $E_{A}$ of $4 \mathrm{kcal} / \mathrm{mol}$ and is inhibitable with pCMBS suggests that protons are passing through the water channel and not some other portion of the vesicle membrane. Reports of differing sensitivities of toad bladder WCV $P_{\mathrm{H}^{+}}$to mercurial reagents $(26,27)$ might well be due to differences in vesicle preparation techniques. In addition, it is unclear whether mammalian WCV possess a mercurial-sensitive component of $\mathbf{P}_{\mathrm{H}^{+}}$(34). If $\mathrm{ADH}$ water channels possess significant proton permeability, it might render collecting duct cells vulnerable to acid loads during antidiuresis particularly when urine $\mathrm{pH}$ is low.

In summary, biophysical studies provide evidence that selective water channels appear to be proteins in which sulfhydryl groups play some critical role. The ADH water channel likely contains a narrow transmembrane pore that at some point is less than $2 \AA$ in diameter. Water channels appear to be present at extraordinarily high density in the limiting membranes of WCV.

\section{Attempts to identify, purify, and clone proteins of the $A D H$ water channel}

During the last 5 years, several ion channels and membrane transporters have been identified and cloned using a variety of 
Table II. Criteria for Candidate ADH Water Channel Protein Components

1. Present on the apical surface exclusively during ADH stimulation

2. Removed from the apical membrane during withdrawal of ADH stimulation

3. Present in endocytic vesicles containing ADH water channels (WCV)

4. Consists of integral membrane proteins that bind membrane lipids

5. Spans the lipid bilayer of WCV

6. Constitutes a large proportion of the protein found in WCV

7. Labeled by mercurial reagents under conditions that lead to inhibition of water flow through the ADH water channel

newly developed techniques (reviewed in Montal [50]). Despite these successes, the understanding of the molecular structure of the ADH water channel is at an early stage. Several characteristics of the ADH water channel have prevented application of techniques used successfully to characterize other channels. The combination of the lack of an inhibitor of ADH water flow possessing sufficient specificity and high affinity to allow identification and purification of $\mathrm{ADH}$ water channel proteins from cellular homogenates together with the absence of a cell line maintaining an ADH-responsive water permeability increase after long-term tissue culture has greatly hindered channel isolation.

Recent data suggest that ADH water channel proteins may be cloned by molecular techniques. Two reports $(51,52)$ have provided evidence that water channels can be expressed in Xenopus oocytes. Increases in mercurial-sensitive oocyte cell membrane $P_{f}$ were observed after injection of either toad bladder granular cell (52) or whole kidney (51) mRNA. Alternatively, since ADH water channels are clustered in retrieved vesicles (see above), purification of WCV and systematic identification of likely channel components from among WCV proteins will allow use of traditional protein chemistry and molecular cloning techniques. It is worth noting that the $\mathrm{ADH}$ water "channel" might well be analogous to other channels that consist of multiple proteins which are responsible for overall channel activity $(46,50)$. In this regard, the $\mathrm{ADH}$ water channel may be more of a transmembrane water transport complex rather than a single protein.

On the basis of data reviewed above, it is probable that major protein components of the ADH water channel will possess several unique characteristics allowing for their tentative identification before molecular cloning and expression studies. These criteria are listed on Table II. Below we will summarize recent progress made by our laboratories and collaborators toward this goal.

\section{Characteristics and proteins of $A D H W C V$ from toad urinary bladder}

Several unusual features of the apical membrane of toad urinary bladder granular cells have enabled isolation of WCV and identification of candidate $\mathrm{ADH}$ water channel proteins. Constitutive turnover of granular cell apical membrane is very slow in the absence of $\mathrm{ADH}$ stimulation (6). In contrast, $\mathrm{ADH}$ stimulation causes fusion of large numbers of subapical aggrephores with the apical membrane. Retrieval of water channel-containing membrane is dramatically increased by transepithelial water flow or removal of ADH (36) allowing selective entrap- ment of fluid phase markers within the lumens of WCV (2427) that possess large numbers of functional water channels.

Since ADH stimulation inserts water channels into the granular cell apical membrane, comparison of exposed apical membrane proteins present on water-impermeable unstimulated bladders to those present under $\mathrm{ADH}$-stimulated conditions would begin to define ADH water channel protein candidates. We (53) and others (54) have performed detailed comparisons using membrane-impermeant probes that covalently label exposed protein domains. Fractionation of these labeled proteins by SDS-PAGE has identified bands of 55, 53, 17, 15, and $7 \mathrm{kD}$ as appearing exclusively on the surface of ADH-stimulated water-permeable bladders. Furthermore, sequestration of a lactoperoxidase membrane iodination mixture within retrieved WCV in intact living cells results in the ${ }^{125} \mathrm{I}$ labeling of the same bands of 55, 53, 17, 15, and $7 \mathrm{kD}$ (53). To further identify the protein bands that are likely parts of the water channel, we have examined the ability of these proteins to interact with lipid using the Triton X-114 partitioning analysis. These studies (35) demonstrate that the 55-, 53-, and 17-kD proteins are integral membrane proteins (Fig. 2). Thus, these integral membrane proteins are inserted into the apical membrane during $\mathrm{ADH}$ stimulation and returned into vesicles when it is withdrawn. It is highly likely that some of these proteins form part of the ADH water channel.

We have purified toad bladder WCV using either a density shift protocol (28) or flow cytometry vesicle sorting (55) and characterized their membrane proteins. Purified WCV are composed of 12-15 protein bands as determined by SDS-PAGE (Fig. $3 \mathrm{D}$ ). Two proteins of 55 and $53 \mathrm{kD}$ that are present in 2:1 ratio account for approximately one half of all vesicle protein. These data would appear to agree with the suggestion that large numbers of water channels would have to exist in WCV membranes based on a comparison of retrieved vesicle $P_{f}$ to that of other water channel-containing membrane (see above). A combination of antibody and peptide mapping data (56) also suggests that the $17-\mathrm{kD}$ WCV integral membrane protein is a fragment of these 55 and $53 \mathrm{kD}$ proteins. These $55-, 53-$, and $17-\mathrm{kD}$ WCV proteins are identical to those candidate ADH water channel proteins identified by studies using membrane impermeant probes to label apical membrane. Use of similar labeling techniques to analyze the protein topography of WCV has demonstrated that the 55-, 53-, and a $46-\mathrm{kD}$ protein span the lipid bilayer of water channel containing vesicles (35).

The 55- and 53-kD proteins are likely components of the ADH water channel

Our most recent work (57) has attempted to link directly modification of these proteins with inhibition of vesicle $P_{f}$. Fluorescein mercuric acetate (FMA), previously shown to inhibit ADH water flow in intact epithelia (16), inhibits WCV $P_{f}$ by $77 \%$ in a manner similar to that exhibited by p-CMBS $(26,57)$. The fluorescein moiety of FMA is recognized by a rabbit antifluorescein antisera. We have probed western blots of WCV proteins to identify those covalently labeled by FMA. These studies show that only four to five protein bands, including the 55- and 53-kD proteins, are FMA-labeled under conditions that inhibit vesicle $P_{f}$.

In summary, the data presented above show that the 55and $53-\mathrm{kD}$ proteins fulfill all the criteria anticipated for protein components of the ADH water channel as listed in Table II. Taken together, these data strongly support the hypothesis that 
Protein Composition

SDS-PAGE Protein Bands (kD)

WCV

Lumen

\section{WCV membrane}

Cell

$100^{\circ} \quad$ (Multiple Species)

86 (Multiple Species)

60 (Integral)

$55^{* 0}$ (Integral)

$53^{* 0}$ (Integral)

46 (Integral)

$38^{\circ}$ (Peripheral)

35 (Integral)

32 (Integral)

30 (Integral)

$17^{*}$ (Integral)

15.5 (Peripheral)

$15^{*}$ (Peripheral)

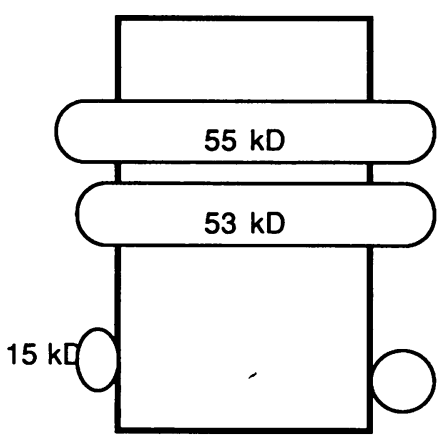

$15.5 \mathrm{kD}$
Figure 2. Composition and topography of membrane proteins in purified ADH WCV from toad bladder granular cells. Left: Purified WCV were separated by SDS-PAGE as described in reference 35. Protein bands that contain multiple proteins of similar molecular mass are designated as "multiple"; otherwise individual proteins are labeled either integral or peripheral based on their behavior in the Triton X-114 partitioning assay and, in some cases, in vectorial labeling assays using membrane impermeant reagents (35). Integral membrane proteins possess lipid binding domains and are embedded in the vesicle lipid bilayer. In contrast, peripheral membrane proteins are merely attached to

the vesicle membrane. ${ }^{* 125}$ I-labeled exclusively on the apical surface of ADH-stimulated toad bladders. ${ }^{\circ}$ Labeled by FMA under conditions where WCV $\mathrm{P}_{\mathrm{f}}$ is inhibited. Right: Topography of several vesicle membrane proteins as determined by vectorial labeling experiments. Since the 55- and 53-kD proteins span the lipid bilayer of water permeable vesicles, they could form a transmembrane aqueous pore.

the 55- and 53-kD proteins are components of the $\mathrm{ADH}$ water channel.

\section{Future directions}

Development of antibodies or cDNA probes to ADH water channel protein components will allow exploration of several important questions. A combination of molecular cloning, protein structural analysis, and reconstitution/expression efforts will begin to define the structure and function of this unique channel at a molecular level including its interactions with lipids of vesicles and the apical membrane. This is equally true for both the amphibian as well as mammalian ADH water
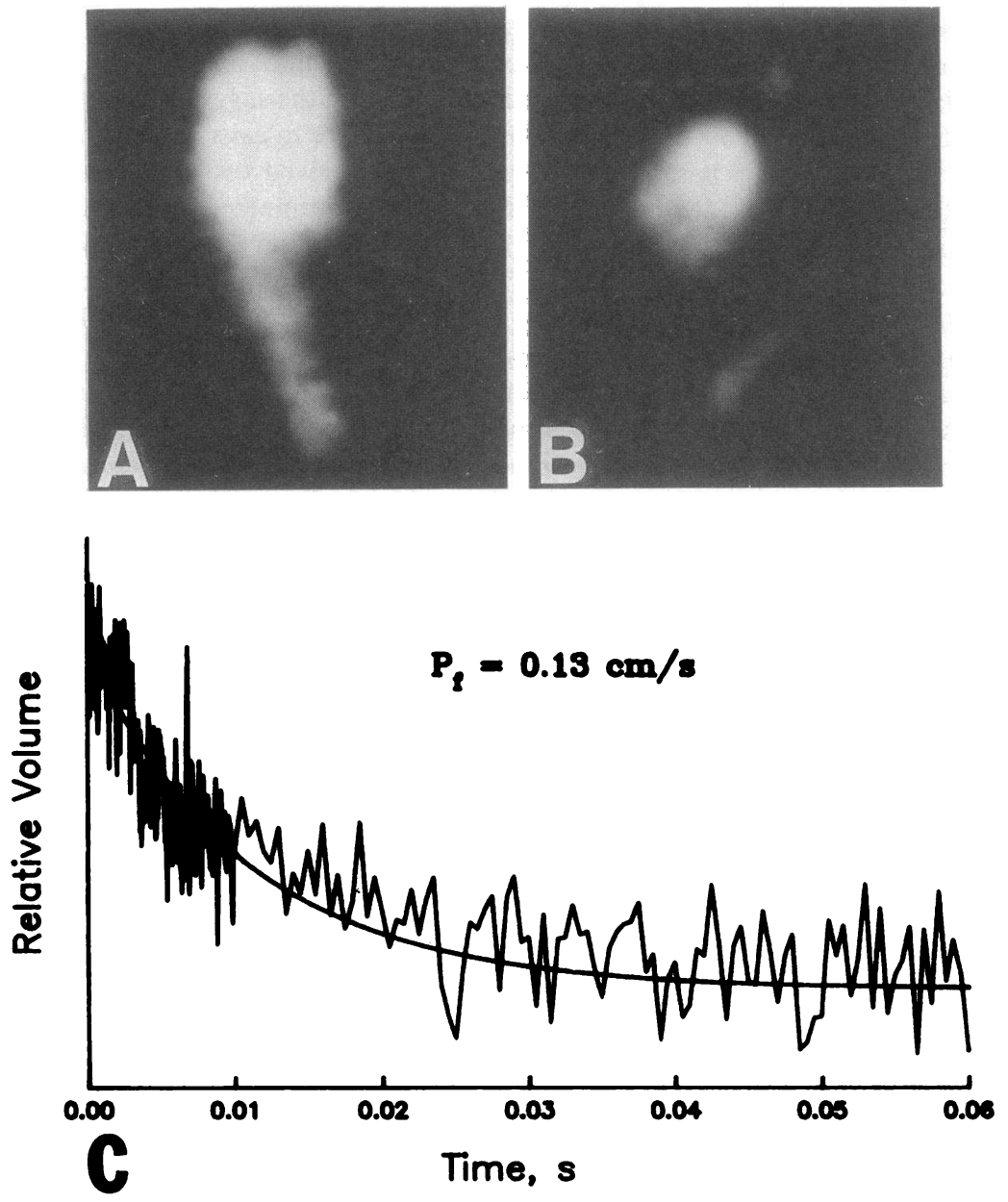

Figure 3. Trafficking, water permeability, and protein composition of vesicles containing ADH water channels from toad urinary bladder. $(A$ and $B$ ) Confocal microscopy images of endocytic vesicles in living toad bladder cells. In $A$, a tubular-shaped vesicle appears to be fused with a large, spherical vesicle. $B$ illustrates the same cellular region imaged $10 \mathrm{~min}$ later. The tubular vesicle is detached and moving away from the spherical structure. (C) A tracing of vesicle volume changes measured by stopped flow fluorimetry used to determine the osmotic water permeability $\left(P_{f}\right)$ of ADH WCV. (D) SDSPAGE gel showing the protein composition of the same vesicles as assayed in $C$ after their purification. Two arrowheads point to candidate ADH water channel proteins of 55 and $53 \mathrm{kD}$. 
channel. Intertwined with this research effort is work on the components that make the apical membranes of unstimulated ADH-responsive epithelial cells relatively impermeable to water. Several other important questions including the synthesis, modification, intracellular processing, and possible recycling of water channel components require study. More effcient purification of aggrephores and their components should enhance studies of the mechanism and control of the translocation of aggrephores and WCV. Present data suggest that water channels may also exist in the renal proximal tubule, human erythrocyte and perhaps the basolateral membrane of ADH-responsive cells (reviewed in detail in Verkman [58]). The relationship between these putative water channels and the ADH water channel remains to be tested.

\section{Acknowledgments}

The authors wish to thank Drs. J. Handler, B. Brenner, S. Hebert, J. B. Wade, and E. Grossman for their critical reading of our manuscript.

This work was supported in part by National Institutes of Health grants DK 38874, 37317, and 38690. Drs. Harris and Strange are recipients of Established Investigator Awards from the American Heart Association. Dr. Zeidel has a Department of Veterans Affairs Research Associate Career Development Award.

\section{References}

1. Marshall, E. K., and H. W. Smith. 1930. Comparative physiology of vertebrate kidney. Biol. Bull. 59:135-153.

2. Knepper, M. A., and F. C. Rector, Jr. 1991. Urinary concentration and dilution. In The Kidney. B. M. Brenner and F. C. Rector, Jr. W. B. Saunders Co. Philadelphia. 445-482.

3. Finkelstein, A. 1986. Water Movement through Lipid Bilayers, Pores and Plasma Membranes: Theory and Reality. John Wiley \& Sons, Inc., New York.

4. Strange, K., and K. R. Spring. 1987. Cell membrane water permeability of rabbit cortical collecting duct. J. Membr. Biol. 96:27-43.

5. Flamion, B., and K. R. Spring. 1990. Water permeability of apical and basolateral cell membranes of rat inner medullary collecting duct. Am. J. Physiol. 259:F986-F999.

6. Harris, H. W., and J. S. Handler. 1988. The role of membrane turnover in the water permeability response to antidiuretic hormone. J. Membr. Biol. 103:207-215.

7. Hebert, S. C., and T. E. Andreoli. 1982. Water movement across the mammalian cortical collecting duct. Kidney Int. 22:526-535.

8. Zeidel, M. L., E. Grossman, and H. W. Harris. 1991. Permeability properties of highly purified apical membranes of toad bladder granular cells. Clin. Res. 39:223A. (Abstr.)

9. Kikeri, D., A. Sun, M. L. Zeidel, and S. C. Hebert. 1989. Cell membranes impermeable to $\mathrm{NH}_{3}$. Nature (Lond.). 339:478-480.

10. Fettiplace, R., and D. A. Haydon. 1980. Water permeability of lipid membranes. Physiol. Rev. 60:510-550.

11. Macey, R. I. 1984. Transport of water and urea in red blood cells. Am. J. Physiol. 246:C195-C203.

12. Van Meer, G. 1990. Biosynthetic lipid traffic in animal eukaryotes. Annu. Rev. Cell Biol. 5:247-275.

13. Breyer, M. D., H. R. Jacobson, and R. L. Hebert. 1990. Cellular mechanisms of prostaglandin $E_{2}$ and vasopressin interactions in the collecting duct. Kidney Int. 38:618-624.

14. Reif, M. C., S. L. Troutman, and J. A. Schafer. 1984. Sustained response to vasopressin in isolated rat cortical collecting tubule. Kidney Int. 26:725-732. 15. Kachadorian, W. A., J. Muller, S. W. Rudich, and V. A. Discala. 1979. Temperature dependence of ADH-induced water flow and intramembranous particle aggregates in toad bladder. Science (Wash. DC). 205:910-913.

16. Hoch, B., P. C. Gorfein, D. Linzer, M. J. Fusco, and S. D. Levine. 1989. Mercurial reagents inhibit flow through ADH-induced water channels in toad bladder. Am. J. Physiol. 256:F948-F953.

17. Levine, S. D., M. Jacoby, and A. Finkelstein. 1984. The water permeability of toad urinary bladder. II. The value of $\mathrm{Pf} / \mathrm{Pd}(w)$ for the antidiuretic hormone-induced water permeation pathway. J. Gen. Physiol. 83:543-561.

18. Wade, J. B., D. L. Stetson, and S. A. Lewis. 1981. ADH action: evidence for a membrane shuttle mechanism. Ann. NY Acad. Sci. 372:106-117.

19. Humbert, F., R. Montesano, A. Grosso, R. C. DeSousa, and L. Orci. 1977. Particle aggregates in plasma and intracellular membranes of toad bladder (granular cell). Experientia (Basel). 33:1364-1367.
20. Muller, J., and W. A. Kachadorian. 1984. Aggregate-carrying membranes during ADH stimulation and washout in toad bladder. Am. J. Physiol. 247:C90C98.

21. Palmer, L. G., and M. Lorenzen. 1983. Antidiuretic hormone dependent membrane capacitance and water permeability in the toad urinary bladder. $\mathrm{Am}$. J. Physiol. 24:F195-F204.

22. Masur, S., S. Cooper, and M. S. Rubin. 1984. Effect of an osmotic gradient on ADH-induced endocytosis and hydroosmosis in the toad urinary bladder. $\mathrm{Am}$. J. Physiol. 247:F370-F379.

23. Coleman, R. A., H. W. Harris, Jr., and J. B. Wade. 1987. Visualization of endocytosed markers in freeze fracture studies of toad urinary bladder. J. Histo chem. Cytochem. 35:1405-1414.

24. Harris, H. W., J. S. Handler, and R. Blumenthal. 1990. Apical membrane vesicles of ADH-stimulated toad bladder are highly water permeable. Am. J. Physiol. 258:F237-F243.

25. Shi, L.-B., and A. S. Verkman. 1989. Very high water permeability in vasopressin-dependent endocytic vesicles from toad bladder granular cells. $J$. Gen. Physiol. 94:1101-1115.

26. Harris, H. W., D. Kikeri, A. Janoshazi, A. K. Solomon, and M. L. Zeidel. 1990. High proton flux through membranes containing antidiuretic hormone water channels. Am. J. Physiol. 259:F366-F371.

27. Shi, L.-B., D. Brown, and A. S. Verkman. 1990. Water, proton and urea transport in toad bladder endosomes that contain the vasopressin-sensitive water channel. J. Gen. Physiol. 95:941-960.

28. Harris, H. W., H. R. Murphy, M. C. Willingham, and J. S. Handler. 1987. Isolation and characterization of specialized regions of toad urinary bladder apical plasma membrane involved in the water permeability response to antidiuretic hormone. J. Membr. Biol. 96:175-186.

29. Verkman, A. S., W. I. Lencer, D. Brown, and D. A. Ausiello. 1988. Endosomes from kidney collecting tubule cells contain the vasopressin-sensitive water channel. Nature (Lond.). 333:268-269.

30. Brown, D., S. Gluck, and J. Hartwig. 1987. Structure of the novel membrane-coating material in proton-secreting epithelial cells and identification as an $\mathrm{H}^{+}$ATPase. J. Cell Biol. 105:1637-1648.

31. Bacskai, B. J., and P. A. Friedman. 1990. Parathyroid hormone activates apical membrane calcium channels in cultured distal kidney cells. Kidney Int. 37:202a. (Abstr.)

32. Ernst, S. A., and T. R. Kleyman. 1991. Immunolocalization of the $\mathrm{Na}$ channel in A6 cells. J. Am. Soc. Nephrol. 1:717a. (Abstr.)

33. Slot, J. W., H. J. Geuze, S. Gigengack, G. E. Lienhard, and D. E. James. 1991. Immuno-localization of the insulin regulatable glucose transporter (Glut 4) in brown adipose tissue of the rat. J. Cell Biol. In press.

34. Lencer, W. I., A. S. Verkman, M. A. Arnout, D. A. Ausiello, and D. Brown. 1990. Endocytic vesicles from renal papilla which retrieve the vasopressin-sensitive water channel do not contain a functional $\mathrm{H}^{+}$ATPase. J. Cell Biol. 111:379-389.

35. Harris, H. W., M. L. Zeidel, and C. Hosselet. 1991. Quantitation and topography of membrane proteins in highly water permeable vesicles from ADHstimulated toad bladder. Am. J. Physiol. In press.

36. Harris, H. W., J. B. Wade, and J. S. Handler. 1986. Transepithelial water flow regulates apical membrane retrieval in antidiuretic hormone-stimulated toad urinary bladder. J. Clin. Invest. 78:703-712.

37. Botelho, B., H. W. Harris, M. Zeidel, and K. Strange. 1991. Transepithelial water flow is not necessary to produce retrieval of ADH water channel containing vesicles in toad urinary bladder. FASEB (Fed. Am. Soc. Exp. Biol.) J. 5:4287a. (Abstr.)

38. Harris, H. W. 1991. Antidiuretic hormone modulates membrane phosphoproteins in toad urinary bladder and retrieved water channel containing apical membrane vesicles. J. Am. Soc. Nephrol. 1:1114-1121.

39. Ding, G., N. Franki, J. Bourguet, and R. M. Hays. 1988. Role of vesicular transport in ADH-stimulated aggregate delivery. Am. J. Physiol. 255:C641C652.

40. Coleman, R. A., and J. B. Wade. 1990. Endosomal sorting of particle aggregates and fluid-phase markers following ADH reversal in toad bladder. $J$. Am. Soc. Nephrol. 1:673a. (Abstr.)

41. Zeidel, M. L., E. Grossman, and H. W. Harris. 1990. Fate of antidiuretic hormone water channel membrane after endocytosis from the apical membrane of toad urinary bladder granular cells. J. Am. Soc. Nephrol. 1:680a. (Abstr.)

42. Brown, D., and L. Orci. 1983. Vasopressin stimulates formation of coated pits in rat kidney collecting ducts. Nature (Lond.). 302:253-255.

43. Strange, K., M. C. Willingham, J. S. Handler, and H. W. Harris, Jr. 1988. Apical membrane endocytosis via coated pits is stimulated by removal of antidiuretic hormone from isolated, perfused rabbit cortical collecting tubule. $J$. Membr. Biol. 103:17-28.

44. Pearl, M., and A. Taylor. 1985. Role of the cytoskeleton in the control of transcellular water flow by vasopressin in amphibian urinary bladder. Biol. Cell. 55:163-172.

45. Vale, R. D. 1987. Intracellular transport using microtubule-based motors. Annu. Rev. Cell Biol. 3:347-378. 
46. McCarthy, M. P., J. P. Earnest, E. F. Young, S. Choe, and R. M. Stroud. 1986. The molecular neurobiology of the acetylcholine receptor. Annu. Rev. Neurosci. 9:383-413.

47. Ibarra, C., P. Ripoche, and J. Bourguet. 1989. Effects of pCMBS and FMA on the water and small solute permeabilities in frog urinary bladder. J. Membr. Biol. 110:586-594.

48. Lear, J. D., Z. R. Wasserman, and W. F. DeGrado. 1988. Synthetic amphiphilic peptide models for protein ion channels. Science (Wash. DC). 240:1177-1181.

49. Gluck, S., and Q. Al-Awqati. 1980. Vasopressin increases water permeability by inducing pores. Nature (Lond.). 284:631-632.

50. Montal, M. 1990. Molecular anatomy and molecular design of channel proteins. FASEB (Fed. Am. Soc. Exp. Biol.) J. 4:2623-2635.

51. Milovanovic, S., M. F. Chao, G. Frindt, and E. E. Windhager. 1989. FASEB (Fed. Am. Soc. Exp. Biol.) J. 3:A997a. (Abstr.)

52. Zhang, R., and A.S. Verkman. 1991. Water and urea permeability properties of Xenopus oocytes: expression of mRNA from toad bladder. Am. J. Physiol. 000:C26-C34.
53. Harris, H. W., J. B. Wade, and J. S. Handler 1988. Identification of specific apical membrane polypeptides associated with the antidiuretic hormoneelicited water permeable increase in toad urinary bladder. Proc. Nat. Acad. Sci. USA. 85:1942-1946.

54. Valenti, G., L. Guerra, V. Cassavola, and M. Svelto. 1990. Selective labeling of the proteins involved in the hydroosmotic response to $\mathrm{ADH}$ in frog urinary epithelia. Biol. Cell. 67:115-122.

55. Grossman, E., T. Hammond, H. W. Harris, and M. L. Zeidel. 1990. Purification of ADH-regulated water channel containing vesicles from toad urinary bladder using flow cytometry. J. Am. Soc. Nephrol. 1:674a. (Abstr.)

56. Harris, H. W., L. Guay-Woodford, and C. Hosselet. 1990. Mouse kidney possess proteins of similar structure to integral membrane proteins of toad bladder aggrephores. Kidney Int. 37:231a. (Abstr.)

57. Harris, H. W., C. Hosselet, and M. L. Zeidel. 1991. Identification of candidate antidiuretic hormone water channel proteins using fluorescein mercuric acetate. Clin. Res. 39:192A. (Abstr.)

58. Verkman, A. S. 1989. Mechanisms and regulation of water permeability in renal epithelia. Am. J. Physiol. 257:C837-C850. 